\title{
Stable and Coordinating Contracts for a Supply Chain with Multiple Risk-Averse Suppliers
}

\author{
Xin Chen \\ Department of Industrial and Enterprise Systems Engineering, University of Illinois at Urbana-Champaign, Illinois 61820, USA, \\ xinchen@illinois.edu \\ Stephen Shum \\ College of Business, City University of Hong Kong, Kowloon Tong, Hong Kong, swhshum@cityu.edu.hk
}

\author{
David Simchi-Levi \\ Engineering Systems Division, Department of Civil and Environmental Engineering and the Operation Research Center, Massachusetts \\ Institute of Technology, Massachusetts 02139-4307, USA, dslevi@mit.edu
}

\begin{abstract}
$\mathrm{W}$ e analyze a decentralized supply chain with a single risk-averse retailer and multiple risk-averse suppliers under a Conditional Value at Risk objective. We define coordinating contracts and show that the supply chain is coordinated only when the least risk-averse agent bears the entire risk and the lowest-cost supplier handles all production. However, due to competition, not all coordinating contracts are stable. Thus, we introduce the notion of contract core, which reflects the agents' "bargaining power" and restricts the set of coordinating contracts to a subset which is "credible." We also study the concept of contract equilibrium, which helps to characterize contracts that are immune to opportunistic renegotiation. We show that, the concept of contract core imposes conditions on the share of profit among different agents, while the concept of contract equilibrium provide conditions on how the payment changes with the order quantity.
\end{abstract}

Key words: supply chain contracts; risk aversion; quantity discount; consignment

History: Received: October 2010; Accepted: December 2012 by Panos Kouvelis after 2 revisions.

\section{Introduction}

Supply chain contracts have received a lot of attention in the academic community and in the industry. A lot of research focuses on mechanism design to provide incentives that induce different agents in the supply chain to achieve global efficiency. That is, the goal is to identify contracts that "coordinate," or align the interest of the different agents with the objective of the supply chain. Unfortunately, a coordinating contract may not be stable, in the sense that some rational decision maker(s) may have incentives to deviate from this contract and hence will not agree to it. For instance, it is obvious that any stable coordinating contract must generate non-negative expected profits for both the supplier and the retailer. However, identifying stable coordinating contracts may not be so simple in other cases, especially when there are competing agents.

Consider two risk-neutral suppliers selling the same product to a risk-neutral retailer. The per unit production costs of supplier 1 and supplier 2 are $\$ 8$ and $\$ 9$, respectively. The retailer sells at $\$ 20$ per unit and faces an uncertain demand uniformly distributed between 800 and 1800. As supplier 1 has a lower pro- duction cost, system optimality is achieved when only supplier 1 produces, and the system optimal production quantity is 1400 units. Now consider the following contract profile; call it Contract Profile $\mathcal{A}$ : a buyback contract between the retailer and supplier 1 with a wholesale price of $\$ 18.8$ and a buyback price of $\$ 18$ and a wholesale contract of $\$ 19$ per piece between the retailer and supplier 2. Under this contract profile, the retailer's optimal decision is to order 1400 units from supplier 1 and order nothing from supplier 2 . Hence, Contract Profile $\mathcal{A}$ is coordinating, and the expected profits of supplier 1, supplier 2, and the retailer under this contract profile are $\$ 11,880, \$ 0$, and $\$ 1320$, respectively.

However, we argue that Contract Profile $\mathcal{A}$ is not stable, because supplier 2 and the retailer are able to get a total expected profit of more than $\$ 1320$ even without working with supplier 1 . Thus, they can come to an agreement which gives both of them a total expected profit of higher than $\$ 1320$. This higher expected profit is guaranteed regardless of the action of supplier 1, that is, even when supplier 1 refuses to supply to the retailer anymore. Therefore, one requirement for a stable contract is that every subset 
of agents should get more than what they can get on their own without working with the other agents. This requirement can be satisfied by adding to Contract Profile $\mathcal{A}$ a side payment from supplier 1 to the retailer. Yet, this side payment does not make Contract Profile $\mathcal{A}$ stable. The reason is that, after the retailer signs the contract with supplier 1 , supplier 2 can offer to sell at a price of less than $\$ 18$. The retailer can then enjoy a higher expected profit by, for example, ordering 800 units from supplier 2 and 600 units from supplier 1. Hence, the retailer will accept the new deal. Supplier 2 also gains from the new deal as it now enjoys a positive expected profit. Thus, a second requirement for a contract to be stable is that no two agents can mutually benefit by changing the contract between them after finalizing the contract terms with other agents. As we see later on, these two requirements are respectively guaranteed by the contract core and contract equilibrium.

Our objective in this study is to introduce an approach to identify coordinating contracts that are stable in supply chains with competing agents. The decisions of different agents (including the retailer and the suppliers) are optimal when they achieve Pareto optimality. We call a contract profile coordinating if it induces optimal decisions in a Nash equilibrium. To identify coordinating contracts that are stable, we introduce the notions of contract core and contract equilibrium. The notion of the contract core is based on the concept of core in cooperative game theory. A contract is in the contract core if every coalition of agents achieves a total objective no less than the value they can achieve by not contracting with agents outside the coalition. On the other hand, the notion of contract equilibrium was introduced by Crémer and Riordan (1987). It follows a non-cooperative approach to study a set of bilateral contracts. In particular, a set of bilateral contracts between a retailer and its many suppliers forms a contract equilibrium if there is no incentive for any supplier-retailer pair to change their contract terms, given the contracts between the supplier and other retailers.

We illustrate the concepts of coordinating contract, contract core, and contract equilibrium in a supply chain with multiple suppliers and a single retailer, where the suppliers and the retailer may be risk neutral or risk averse. While the owner or stockholders of a firm may not prefer risk-averse decisions in the firm, it has been pointed out that managers have incentives to reduce the risk of the firm to avoid losing their employment (Amihud and Lev 1981, Jensen and Meckling 1976). There is also empirical evidence on the relation between executive stock options and the risk attitudes of managerial decision making (Rajgopal and Shevlin
2002). We capture this kind of risk-averse behavior of each agent employing the concept of Conditional Value at Risk (CVaR), which is gaining popularity in the finance industry as a risk measure.

We show that optimality is achieved when the lowest-cost supplier produces and the least risk-averse supplier bears entire risks in the supply chain. Different types of contracts such as quantity discount and consignment contracts can be used to coordinate in different cases. In particular, when a supplier who does not have the lowest cost is the least risk-averse agent in the whole supply chain, the coordinating contract is equivalent to this least risk-averse supplier purchasing from the lowest-cost supplier under a quantity discount contract and then selling to the retailer under a consignment contract. Thus, a supplier has an active role in the supply chain if he has the lowest cost or he is the least risk-averse agent.

Other suppliers, who do not have the lowest cost and are not the least risk averse, do not have an active role and receive zero objective under the coordinated contract. Thus, these suppliers will try to offer alternative deals to the supplier. Using the concepts of contract core and contract equilibrium, we identify among all coordinating contracts the ones that are stable. The contract of the contract core allows us to endogenously evaluate the appropriate profit shares of different agents which reflect their bargaining powers. The concept of contract equilibrium imposes conditions on how the payment changes with the order quantity, which we call the contract structure. Thus, using these two concepts we identify coordinating contracts that are stable in terms of share of objectives and contract structure. To the best of our knowledge, this is the first study which attempts to design coordinating contracts that have a stable share of objectives and contract structure. On the practical side, how to design contracts that achieve efficiency is an important issue for managers. However, efficiency can be disrupted if a supply chain partner negotiates a new contract with another party. It is important to provide insights on how to design contracts that can achieve efficiency and guard against renegotiation. On the theoretical side, our approach of adopting the contract core and contract equilibrium allows detailed analysis of the stability of the contract structure while abstracting away from the details of the negotiation process. This approach may find applicability in other contract design problems.

The organization of this study is as follows. Section 2 reviews the literature. In section 3, we describe our model. We study coordination in section 4 and the stability of coordinating contracts in section 5. Finally, we conclude in section 6 . All proofs are relegated to the Online Appendix. 


\section{Literature Review}

Our study is closely related to the stream of literature on supply chain contracting with competing risk-averse agents. For example, Agrawal and Seshadri (2000) study a supply chain with one supplier and multiple independent non-competing retailers where every agent maximizes the expectation of a concave utility function. They show that performance of the supply chain is improved if there exists a risk-neutral intermediate agent taking all the risks. Spulber (1985) studies a similar model with a single supplier selling to multiple non-competing retailers. He shows that in the setting with a risk-neutral agent, the risk-neutral agent takes all the risks to achieve supply chain coordination. However, if no risk-neutral agent exists in the system, all agents share the risks. Gan et al. (2004) study a supply chain with a single supplier and a single retailer and model risk aversion by the expected exponential utility objective and the mean-variance objective. They show that the agents share risks under coordinating contracts in both cases. All these studies show that Pareto optimality is achieved when the agents share the risks unless a risk-neutral agent exists in the system. Different from the aforementioned studies, we show that, even when the agents are risk averse, Pareto optimality is achieved when the least risk-averse agent bears all the risk. In addition, we consider the stability of coordinating contracts in this study.

Recently, the notion of stability has received some attention in the operations literature. Different methods and notions related to stability have been used to study supply chains, such as the Nash bargaining solution (Gurnani and Shi 2006, Nagarajan and Bassok 2002), farsighted stability (Nagarajan and Sosic 2007, 2009), and analysis of a bargain game similar to Rubinstein's model (Bernstein and Marx 2006). However, certain properties of these notions and methodologies present difficulties in a detailed analysis of the contract structure. For example, in the Nash bargaining solution, the sequence of negotiations has a huge impact on the outcomes; analysis using farsighted stability normally assumes that all players in a coalition act like a single decision player. Results from analyses similar to Rubinstein's model depend heavily on details of the bargaining process and usually restrict the types of contracts proposed during negotiations. Thus, these studies focus on characterizing the equilibrium market outcome (such as stable coalition structure or stable pricing cartel) or on analyzing the post-negotiation profit given a contract structure. On the other hand, our adoption of the concepts of contract core and contract equilibrium allows us to study the contract structure in detail and focus on designing contracts that achieve optimality, stability in objectives share, and contract structure.

While the observation that coordinating contracts may not be stable is not new, there has been little attention on how to design coordinating contracts that are stable. Thus, an important contribution of this study is to investigate this distinctive feature of stability which has generally been overlooked in existing literature on supply chain coordination (see Cachon 2003 for review). A study with similar focus is Anupindi et al. (2001). They study a two-period model in which different retailers make inventory decisions non-cooperatively in the first period and then they decide cooperatively how to share inventory in the second period. Using the concept of the core, they identify contracts such that all agents cooperatively allocate their stocks "for a given pattern of stocking positions by the various agents, and a given demand pattern" after signing the contracts. In this study, our focus is to provide necessary conditions such that all agents will sign the contracts, and the concepts of the contract core and contract equilibrium present approaches to achieve that.

Economics literature has also paid attention to stable outcomes when an agent engages in bilateral contracts with multiple competing agents. For example, some studies (such as de Fontenay and Gans 2005, Hart and Tirole 1990) model the negotiation process as an extensive form game and analyze the subgame perfect equilibrium. They focus on incentives for vertical integration rather than the design of efficient contracts. Crémer and Riordan (1987) first introduced the notion of contract equilibrium to study the stability of efficient contracts. They study a hierarchy with one upstream party serving multiple downstream agents, where there is no competition or uncertainty but the production cost is a general function. They identify conditions under which there exist contract equilibria that coordinate the system. O'Brien and Shaffer (1992) show that quantity-based contracts do not form contract equilibrium with the system optimal decisions under downstream competition when there is no uncertainty. The focus of these latter two studies is similar to ours. We show that quantity-based contracts may form contract equilibrium with system optimal decisions under upstream competition when there is demand uncertainty. In addition, we introduce the concept of contract core to further eliminate certain contracts that are not stable.

\section{The Model}

Consider a supply chain with a single retailer and multiple suppliers where the agents are risk averse. The retailer faces an uncertain demand $D$ with a 
continuous distribution function $F(\cdot)$. Before the realization of the demand, the retailer orders a quantity $q_{r_{i}}$ from each supplier $i$. Each supplier $i$ then produces and delivers a quantity $q_{s_{i}} \leq q_{r_{i}}$, also before demand is realized. Let $\boldsymbol{q}_{r}=\left(q_{r_{1}}, \ldots, q_{r_{n}}\right)$ and $q_{s}=\left(q_{s_{1}}, \ldots, q_{s_{n}}\right)$. Notice that $\boldsymbol{q}_{s} \leq \boldsymbol{q}_{r}$. The selling price of the retailer is $p$ per unit, and the production cost of supplier $i$ is $c_{i}$ per unit. Without loss of generality we assume that $c_{1} \leq c_{2} \leq \cdots \leq c_{n}$. We assume the product has zero salvage value, but we expect the qualitative insights to hold even in the case of a positive salvage value. Finally, before making the ordering decisions, the retailer and each of the suppliers agree to a payment scheme (or contract) denoted as $T_{i}\left(\boldsymbol{q}_{s}, D\right)$, which is the payment from the retailer to supplier $i$. We denote $\boldsymbol{T}=\left(T_{1}\left(\boldsymbol{q}_{\mathbf{s}}, D\right), \ldots, T_{n}\left(\boldsymbol{q}_{\boldsymbol{s}}, D\right)\right)$ as a contract profile, that is, the vector of all payment schemes from the retailer to the suppliers. We focus on contract profiles $T$ such that

1. $\int T_{i}\left(\boldsymbol{q}_{s}, D\right) d F(D)$ exists for all $\boldsymbol{q}_{s}$ and $i$.

2. $T_{i}\left(\boldsymbol{q}_{s}, D\right)$ is either left-continuous or right-continuous for all $q_{s}$ and $i$.

Let $\mathcal{T}$ be the set of all possible contract profiles satisfying these assumptions.

The decisions of the agents depend not only on the contract profile $T$ but also on the objective functions of the agents. As discussed in the Introduction, we use $\mathrm{CVaR}$ as the objective function of each agent to better capture the behavior of managerial decision making. To define CVaR, we follow Rockafellar and Uryasev (2002). First, observe that it is closely related to Value at Risk (VaR; see Dowd 1998, Duffie and Pan 1997, Jorion 1997), which is defined for a given random variable $\tilde{z}$ and a scalar $\eta \in[0,1]$ as the $1-\eta$ percentile of the random variable $\tilde{z}$, that is, $\operatorname{VaR}_{\eta}(\tilde{z})=\sup \{z \mid 1-\operatorname{Pr}(\tilde{z} \leq z) \geq \eta\}$. Then, we introduce the $\eta$-head distribution of the random variable $\tilde{z}, \Psi_{\eta}(z)$, as follows:

$$
\Psi_{\eta}(z)= \begin{cases}1, & \text { if } z \geq \operatorname{VaR}_{\eta}(\tilde{z}) \\ \frac{\operatorname{Pr}(\tilde{z} \leq z)}{1-\eta}, & \text { if } z<\operatorname{VaR}_{\eta}(\tilde{z}) .\end{cases}
$$

The $\eta-C V a R$ of the random variable $\tilde{z}$ is then defined as the mean of the $\eta$-head distribution of $\tilde{z}$, that is,

$$
\begin{aligned}
\operatorname{CVaR}_{\eta}(\tilde{z}) & =E_{\Psi_{\eta}}[\tilde{z}] \\
& =\frac{1}{1-\eta} \int_{-\infty}^{z_{\eta}-} z d \operatorname{Pr}(\tilde{z} \leq z)+\left(1-\frac{1-\eta^{\prime}}{1-\eta}\right) z_{\eta},
\end{aligned}
$$

where for simplicity, $z_{\eta}=\operatorname{VaR}_{\eta}(\tilde{z})$ and $\eta \leq \eta^{\prime}=$ $1-\operatorname{Pr}\left(\tilde{z}<z_{\eta}\right)$. We refer to Rockafellar and Uryasev (2002) for more discussions and illustrative figures regarding the concept of $\mathrm{CVaR}$. Observe that the parameter $\eta$ in the definition of $\mathrm{CVaR}$ reflects the degree of risk aversion of the decision maker. It is clear that when $\eta=0, \mathrm{CVaR}_{\eta}(\tilde{z})$ reduces to the expectation of $\tilde{z}$. Further, as $\eta$ increases, the decision maker based on $\mathrm{CVaR}_{\eta}$ objective becomes more risk averse. In addition, $\mathrm{CVaR}_{\eta}$ is superadditive.

Now assume that $\tilde{z}$ is a function of a continuous random variable $\tilde{v} \in V$, that is, $\tilde{z}=g(\tilde{v})$. It is easy to show that

$$
\operatorname{CVaR}_{\eta}(\tilde{z})=E[g(\tilde{v}) \mid \tilde{v} \in \mathcal{V}],
$$

where $\mathcal{V}$ is any subset of $V$ satisfying (a) $\operatorname{Pr}(\tilde{v} \in \mathcal{V})=1-\eta$, and (b) $g\left(\tilde{v}^{\prime}\right) \leq g\left(\tilde{v}^{\prime \prime}\right)$ for all $v^{\prime} \in \mathcal{V}$ and $v^{\prime \prime} \notin \mathcal{V}$.

Given the delivery quantity vector $q_{s}$ and the contract profile $\boldsymbol{T}\left(\boldsymbol{q}_{s}, D\right)$, the profit of the retailer $\pi_{0}$ is given by $\pi_{0}\left(\boldsymbol{q}_{s}, D, \boldsymbol{T}\right)=p \min \left(q_{0}, D\right)-\sum_{i=1}^{n} T_{i}\left(\boldsymbol{q}_{\boldsymbol{s}}, D\right)$ where $q_{0}=\sum_{i=1}^{n} q_{s_{i}}$. The CVaR objective of the retailer, is given by

$$
\begin{aligned}
U_{0}\left(\boldsymbol{q}_{s}, \boldsymbol{T}\right) & =C V a R_{\eta_{0}}\left(\pi_{0}\left(\boldsymbol{q}_{s}, D, \boldsymbol{T}\right)\right) \\
& =E\left[\pi_{0}\left(\boldsymbol{q}_{\boldsymbol{s}}, D, \boldsymbol{T}\right) \mid D \in \mathcal{D}_{0}\right],
\end{aligned}
$$

where $\mathcal{D}_{0}$ is any subset of $\mathbf{R}^{+}$satisfying $\operatorname{Pr}\left(D \in \mathcal{D}_{0}\right)=1-\eta_{0} \quad$ and $\quad \pi_{0}\left(\boldsymbol{q}_{s}, D^{\prime}, \boldsymbol{T}\right) \leq \pi_{0}\left(\boldsymbol{q}_{\boldsymbol{s}}\right.$, $\left.D^{\prime \prime}, T\right)$ for all $D^{\prime} \in \mathcal{D}_{0}$ and $D^{\prime \prime} \notin \mathcal{D}_{0}$. Similarly, supplier $i^{\prime}$ s profit is given by $\pi_{i}\left(\boldsymbol{q}_{s}, D, \boldsymbol{T}\right)=$ $T_{i}\left(\boldsymbol{q}_{\boldsymbol{s}}, D\right)-c_{i} q_{s_{i}}$, and its CVaR objective is given by

$$
\begin{aligned}
U_{i}\left(\boldsymbol{q}_{\boldsymbol{s}}, \boldsymbol{T}\right) & =C V a R_{\eta_{i}}\left(\pi_{i}\left(\boldsymbol{q}_{\boldsymbol{s}}, D, \boldsymbol{T}\right)\right) \\
& =E\left[\pi_{i}\left(\boldsymbol{q}_{\boldsymbol{s}}, D, \boldsymbol{T}\right) \mid D \in \mathcal{D}_{i}\right],
\end{aligned}
$$

where $\mathcal{D}_{i}$ is any subset of $\mathbf{R}^{+}$satisfying $\operatorname{Pr}\left(D \in \mathcal{D}_{i}\right)=1-\eta_{i}$ and $\pi_{i}\left(\boldsymbol{q}_{s}, D^{\prime}, \boldsymbol{T}\right) \leq \pi_{i}\left(\boldsymbol{q}_{s}, D^{\prime \prime}, \boldsymbol{T}\right)$ for all $D^{\prime} \in \mathcal{D}_{i}$ and $D^{\prime \prime} \notin \mathcal{D}_{i}$.

For the simplicity of presentation, we assume that no two suppliers have the same risk-aversion level for the rest of the section, that is, $\eta_{i} \neq \eta_{j}$ whenever $i \neq j$ and $i, j \neq 0$. Most of the results in this section can be extended to the case when two or more suppliers have the same risk-aversion level. Define $m$ as the index of the least risk-averse supplier, that is, $\eta_{m} \leq \eta_{i}$ for all $i>0$. Let $\eta=\min \left(\eta_{0}, \eta_{m}\right)$ be the percentile used for the $\mathrm{CVaR}$ objective of the least risk-averse agent.

We now comment on the use of $\mathrm{CVaR}$ as the risk measure in our model. We first note that although it is common to use an expected concave utility function as a risk measure, firms usually do not have such an explicit form of utility function. Instead, more often than not, firms set aspirations and targets and correspondingly make decisions so as to maximize the probability that aspiration levels or targets can be achieved. For instance, Lanzillotti (1958) interviewed officials from 20 big corporations and concluded that one of the most typical objectives is to achieve a target 
return on investment. In another study, Payne et al. (1980) provide empirical evidence of the need to incorporate aspiration levels in the analysis of risky choice behavior. Payne (2005) argues that theories of how to make risky choices need to take into account measures of the probabilities of a gain and a loss. Unfortunately, the probability that aspiration levels or targets can be achieved lacks the convexity property, which imposes a significant computational challenge in general.

Thus, instead of using the expected utility or the probability of achieving an aspiration level to evaluate the risk of an agent, we use $\mathrm{CVaR}$ as a risk measure in our study for the following reasons. First, CVaR, as will be seen in the next section, focuses on the tail of the distribution. This is consistent with the study of managerial perspective towards risk by Shapira (1994), who points out that managers "claimed to be primarily concerned with the downside of the distribution of outcomes." The study also reveals that "most managers referred to the 'worst possible outcome' in defining risk. Such a definition is reflected by the tail of the distribution..." Second, CVaR is a coherent risk measure and preserves the property of subadditivity (see Artzner et al. 1999). As a consequence, it preserves the convexity property and thus is computationally tractable (see Rockafellar and Uryasev 2000). Third, it is proven that $\mathrm{CVaR}$ provides the best convex approximation to models based on maximizing the probability of achieving an aspiration level (see Chen and Sim 2006). As such, CVaR is gaining popularity as a risk measure in the finance industry (see, for instance, applications in the insurance industry [Embrechts et al. 1997], credit risk evaluations [Bucay and Rosen 1999] and portfolio optimization [Rockafellar and Uryasev 2000]) and it has been observed that $\mathrm{CVaR}$ can be better than other metrics in terms of performance (Topaloglou et al. 2002) and applicability (Mulvey and Erkan 2005). The nice properties of CVaR have also "led many financial institutions to use it as a risk measure internally" (Hull 2007). In addition, CVaR has also been used to study inventory control and supply chain management under risk aversion (see Ahmed et al. 2007, Chen et al. 2009, Tomlin and Wang 2005, and the unpublished version of Chen et al. 2007).

One limitation of $\mathrm{CVaR}$ is that it does not satisfy the independence axiom fundamental in the expected utility theory (Street 2010). The independence axiom suggests that the preference relation of any two lotteries will not change when mixing an additional lottery with each of them. While the independence axiom is intuitive, it has been shown to be inconsistent with experimental observations. The Allais Paradox, for example, is a key example of the systematic violation of the independence axiom (Allais 1953, Gollier 2004).

\section{Optimality and Coordination}

In this section, we identify coordinating contract profiles. A coordinating contract profile is one that achieves supply chain optimality in equilibrium. Thus, before we study coordination, we need to first define what is optimal for the supply chain. When all agents in the supply chain are risk neutral, optimality is defined as the situation when the expected total profit of the supply chain is maximized. However, this definition may not carry over to the case when the agents are risk averse. We follow Gan et al. (2004) to use the concept of Pareto optimality, as this concept is commonly used in economics literature as a measure for social welfare. We demonstrate that, under $\mathrm{CVaR}$ objectives, Pareto optimality is equivalent to maximizing the sum of objectives of all agents.

\section{DEFINITION 1.}

(a) The quantity vectors $q_{r}$ and $q_{s}$ are optimal under contract profile $T(\cdot)$ if

(i) $q_{r} \geq q_{s}$; and

(ii) there do not exist $q_{r}^{\prime}, q_{s^{\prime}}^{\prime}$, and $T^{\prime}(\cdot)$ such that $U_{i}\left(\boldsymbol{q}_{s}^{\prime}, \boldsymbol{T}^{\prime}\right) \geq U_{i}\left(\boldsymbol{q}_{\mathrm{s}}, \boldsymbol{T}\right)$ for all $0 \leq i \leq n$ and $U_{j}\left(\boldsymbol{q}_{s}^{\prime}, \boldsymbol{T}^{\prime}\right)>U_{j}\left(\boldsymbol{q}_{s}, \boldsymbol{T}\right)$ for some $0 \leq j \leq n$.

(b) A contract profile $T(\cdot)$ is said to be coordinating if there exist subgame perfect equilibrium quantity vectors $\boldsymbol{q}_{r}$ and $\boldsymbol{q}_{s}$ that are optimal under $T(\cdot)$.

The first condition in Definition 1(a) requires the suppliers' production quantities to be no more than the retailer's order quantities, and thus guarantees that $q_{r}$ and $q_{s}$ are feasible decisions. The second condition suggests that under the given quantities and contract profile, it is not possible to improve the objective of one agent (which can be the retailer or one of the suppliers) without hurting another agent. Thus, this condition suggests that the given quantities and contract profile achieve Pareto optimality.

Several observations are noteworthy. First, the optimality of a strategy profile depends on the contract profile. An unfortunate consequence is that there may not exist an optimal strategy profile under some contract profiles. This is different from the case of risk-neutral agents where certain production quantity vectors are optimal regardless of the contract profile. Thus, Definition $1(\mathrm{~b})$ suggests that a contract is coordinating 
only if there exist $q_{r}$ and $q_{s}$ that are optimal under the contract.

Second, $q_{r}$ and $q_{s}$ can be optimal even when $q_{r} \neq q_{s}$. This is because our model focuses on voluntary compliance where a supplier can produce less than the quantity ordered by the retailer. Thus, $\boldsymbol{q}_{r}$ has no impact on the objectives of the agents; it only affects the feasibility of $\boldsymbol{q}_{s}$, which directly affects the agents' objectives. The other compliance regime is forced compliances, where a supplier has to deliver the exact quantity ordered by the retailer (see Cachon 2003 for discussion regarding the two compliance regimes). Although our model focuses on voluntary compliance, we try to identify contracts that coordinate the supply chain under both compliance regimes. Thus, we focus on characterizing $q_{s}$ that achieves Pareto optimality and identify contracts where ordering the Pareto-optimal quantities is optimal for the retailer and fulfilling the retailer's order is an equilibrium for the suppliers given that the retailer orders the Pareto-optimal quantities.

Third, as CVaR is a transferrable objective (i.e., if $\sum_{i=0}^{n} U_{i}\left(\boldsymbol{q}_{s}, \boldsymbol{T}\right)=\mathcal{U}$ for some $\boldsymbol{q}_{s}$ and $\boldsymbol{T}$, then for any $\sum_{i=0}^{n} \mathcal{U}_{i}=\mathcal{U}$, there exists $T^{\prime}$ such that $\mathcal{U}_{i}\left(q_{s}, T^{\prime}\right)=\mathcal{U}_{i}$, or equivalently objectives under $\mathrm{CVaR}$ can be "reallocated" arbitrarily among the players using side payments) the Pareto-optimal frontier is the plane where the sum of objectives of all agents is maximized. Thus, identifying optimal quantities and contract profiles is equivalent to maximizing $\sum_{i=0}^{n} U_{i}\left(\boldsymbol{q}_{s}, \boldsymbol{T}\right)$. Lemmas 1 and 2 characterize optimal quantity vectors and contract profiles.

LEMMA 1. If $q_{s}^{*}$ is a supply chain optimal production vector, then $q_{s_{i}}^{*}=0$ for all $i>l$, where $l$ is the largest index of suppliers having the lowest production cost.

The lemma thus suggests that Pareto optimality can be achieved only when all products are produced by the supplier with the lowest production cost, which is intuitive. As pointed out in previous discussion, the optimality of $q_{s}^{*}$ depends on the contract profile. In the next lemma, we first identify contract profiles under which there exists $q_{s}^{*}$ that achieve Pareto optimality under all demand distributions.

Lemma 2. The following conditions are necessary and sufficient for the existence of optimal quantity vector $\boldsymbol{q}_{s}^{*}$ under contract profile $\mathbf{T}(\cdot)$.

(a) For an agent a who is more risk averse than the least risk-averse agent, its $1-\eta$ percentile profit is constant as long as $\eta \geq \eta$, that is, $\operatorname{VaR}_{\eta}\left(\pi_{a}\left(\boldsymbol{q}_{s}^{*}, D, \boldsymbol{T}\right)\right)=\operatorname{VaR}_{\underline{\eta}}\left(\pi_{a}\left(\boldsymbol{q}_{s}^{*}, D, \boldsymbol{T}\right)\right)$ if $\eta \geq \underline{\eta}$ and $\eta_{a}>\underline{\eta}$. (b) For any agent $a, \pi_{a}\left(\boldsymbol{q}_{s}^{*}, \boldsymbol{T}(\cdot), D^{\prime}\right) \leq \pi_{a}\left(\boldsymbol{q}_{s}^{*}, \boldsymbol{T}(\cdot), D^{\prime \prime}\right)$ for all $D^{\prime} \leq \operatorname{VaR}_{\eta}(D) \leq D^{\prime \prime}$ with probability 1. In other words, when the demand falls below the $1-\eta$ percentile of demand, the profit of every agent is below its $1-\eta$ percentile of profit.

In addition, the probability that the demand falls below optimal production quantity $q_{0}^{*}=\sum_{i=1}^{n} q_{s_{i}}^{*}$ is no more than $1-\underline{\eta}$, that is, $P\left[D \leq q_{0}^{*}\right] \leq 1-\underline{\eta}$. Hence, the profit for any agent who is more risk averse than the least risk-averse agent is constant when demand is less than the total production quantity.

Observe that under any practical contract, the profit of every agent should remain constant when demand is higher than the total production quantity. Hence, Lemma 2 implies that, in order to achieve Pareto optimality, the least risk-averse agent will take all the risk while all other agents will receive a payment independent of the realization of the demand.

This observation is very different from the results of similar studies that consider expected concave utility and mean-variance trade-off (Agrawal and Seshadri 2000, Gan et al. 2004, Spulber 1985). These studies show that Pareto optimality is achieved when the agents share the risks unless a risk-neutral agent exists in the system. On the other hand, we find that when the agents consider CVaR, then Pareto optimality can be achieved only when the least risk-averse agent takes all the risks. In fact, our proof implies that the result can be extended to a more general objective function $\mu_{\eta}(\tilde{z})$ (parameterized by a scalar $\eta$ with the interpretation that the larger the $\eta$, the more risk averse the agent) as long as (a) $\mu_{\eta}(\tilde{z})$ is superadditive, that is, $\mu_{\eta}(\tilde{x}+\tilde{y}) \geq \mu_{\eta}(\tilde{x})+\mu_{\eta}(\tilde{y})$, and (b) $\mu_{\eta}(\tilde{z})$ is decreasing in $\eta$. Note that superadditivity is a fundamental requirement for coherent risk measures (as we focus on profit instead of loss, we use superadditivity rather than subadditivity as commonly seen in the coherent risk measure literature). It can be shown that the exponential utility and the mean-variance objective do not satisfy superadditivity.

Due to superadditivity, the CVaR of a portfolio is higher than or equal to the sum of CVaRs of the individual risky assets in the portfolio. In other words, the marginal increase in CVaR by adding one risky asset to an existing portfolio is always no smaller than the $\mathrm{CVaR}$ of the risky asset itself. As the $\mathrm{CVaR}$ of a risky asset for the least risk-averse agent is always higher than that for other agents, transferring risky assets from other agents to the least riskaverse agents always increases the sum of objectives of all agents. However, as the exponential utility and the mean-variance objective are not superadditive, the marginal increase in the expected utility or mean-variance by adding one risky asset to an 
existing portfolio can be smaller than the expected utility or mean-variance of the risky asset itself. Even though the expected utility or mean-variance of a risky asset for the least risk-averse agent is higher than that for other agents, transferring some risky assets from the portfolio of the least risk-averse agent to other agents may sometimes increase the sum of expected utility or mean-variance of all agents. Thus, in this case, it is optimal for different agents to share the risks.

The case where the least risk-averse agent is a supplier that does not have the lowest production cost deserves special attention. In this case, to achieve Pareto optimality, the supplier with the lowest production cost should produce everything, while the least risk-averse supplier should take all the risks and the retailer's profit is independent of the demand. It is clear that this case is equivalent to the following scenario: the least risk-averse supplier purchases products from the lowest-cost supplier while selling the products to the retailer under a consignment contract.

Summarizing results from Lemmas 1 and 2, optimality is achieved when the lowest-cost supplier produces all products and the least risk-averse agents bear all risk. It can be shown that the optimal production quantity in the supply chain is equal to that of a newsvendor having a cost equal to the lowest cost supplier and a level of risk aversion equivalent to the least risk-averse agent. Thus, the optimal production quantity is decreasing in both the cost of the lowestcost supplier and the level of risk aversion of the least between supplier $i$ and the retailer in which the retailer pays $M q_{i}$ to supplier $i$. This payment scheme implies that the retailer will never procure from this supplier.

We start by focusing on a special case.

THEOREM 1. Suppose the retailer has the same level of risk aversion as the least risk-averse supplier and this supplier also has the lowest production cost, that is, $\eta_{0}=\eta_{1}=\underline{\eta}$. Then $T \in \mathcal{T}$ with

(a) $T_{i}\left(\boldsymbol{q}_{s}, D\right)=M q_{s_{i}}$ for all $i \neq 1$, and

(b) $T_{1}\left(q_{s_{1}}, D\right)$ being any contract that coordinates a supply chain with a single risk-neutral supplier and a single risk-neutral retailer is coordinating, that is, $\boldsymbol{q}_{s}^{*}$ is a Nash equilibrium under $\boldsymbol{T}$.

Given the extensive research on coordinating contracts in the risk-neutral single-supplier-single-retailer setting, Theorem 1 implies that many contracts remain coordinating as long as only one supplier needs to be involved to achieve Pareto-optimality and this supplier has the same risk-aversion level as the retailer.

We now propose coordinating contracts for other cases.

THEOREM 2. We consider three cases:

(a) The risk-averse level of the retailer is less than or equal to the risk-averse levels of all suppliers, that is, $\underline{\eta}=\eta_{0} \leq \eta_{i}$ for all $i$. Define $\boldsymbol{T}^{d(\alpha)} \in \mathcal{T}$ as

$$
T_{i}^{d(\alpha)}\left(\boldsymbol{q}_{\boldsymbol{s}}, D\right)= \begin{cases}\alpha p C V a R_{\underline{\eta}}\left(\min \left(q_{s_{1}}, D\right)\right)+(1-\alpha) c_{1} q_{s_{1}}, & \text { if } i=1 \\ M q_{s_{i}}, & \text { if } i \neq 1\end{cases}
$$

risk-averse agent.

Now that we have characterized contract profiles under which some $q_{s}^{*}$ can achieve Pareto optimality, we shall identify among these contract profiles a subset under which the Pareto-optimal $q_{s}^{*}$ is a Nash equilibrium. In other words, we shall identify contract profiles that are coordinating. For this purpose, let $M$ be a large number and consider a payment scheme
Then, $T^{d(\alpha)}$ is a coordinating contract profile for all $0 \leq \alpha \leq 1$. Moreover, $T_{1}^{d}$ is a quantity discount contract.

(b) Suppose the risk-averse level of the retailer is higher than or equal to the level of the least riskaverse supplier, who does not have the lowest production cost, that is, $\underline{\eta}=\eta_{m} \leq \eta_{0}$ but $c_{m} \neq c_{1}$. Define $T^{d c(\alpha)} \in \mathcal{T}$ as

$$
T_{i}^{d c(\alpha)}\left(\boldsymbol{q}_{s}, D\right)= \begin{cases}\alpha p C V a R_{\underline{\eta}}\left(\min \left(q_{s_{1}}, D\right)\right)+(1-\alpha) c_{1} q_{s_{1}}, & \text { if } i=1 ; \\ p \min \left(q_{0}, D\right)-[\alpha+\gamma(1-\alpha)] p C V a R_{\underline{\eta}}\left(\min \left(q_{0}, D\right)\right) & \\ -(1-\gamma)(1-\alpha) c_{1} q_{0}+M q_{s_{m}}, & \text { if } i=m ; \\ M q_{s_{i}}, & \text { otherwise. }\end{cases}
$$


Then, $\boldsymbol{T}^{d c(\alpha)}$ is a coordinating contract profile for all $0 \leq \alpha \leq 1,0 \leq \gamma \leq 1$. Note that here $T_{1}^{d c(\alpha)}$ is a quantity discount contract and $T_{m}^{d c(\alpha)}$ is a consignment contract.

(c) Suppose the supplier with the lowest production cost has the least risk-averse level among all agents, that is, $\eta=\eta_{1} \leq d_{r}$. Define $T^{c(\alpha)} \in \mathcal{T}$ as risk and the ownership of the inventories at the retailer. In this case, the coordinating contracts can be implemented in a way such that supplier $m$ purchases the products from supplier 1 with a quantity discount contract and sells to the retailer using a consignment contract.

In the last case, the lowest cost supplier is also the least risk-averse agent. Then, it is optimal for this

$$
T_{i}^{c(\alpha)}\left(\boldsymbol{q}_{s}, D\right)= \begin{cases}p \min \left(q_{s_{1}}, D\right)-(1-\alpha) p C V a R_{\underline{\underline{\eta}}}\left(\min \left(q_{s_{1}}, D\right)\right)+(1-\alpha) c_{1} q_{s_{1}}, & \text { if } i=1 \\ M q_{s_{i}}, & \text { if } i \neq 1\end{cases}
$$

Then $T^{c(\alpha)}$ is a coordinating contract profile (i.e., $\boldsymbol{q}_{s}^{*}$ is a Nash equilibrium under $\boldsymbol{T}^{c(\alpha)}$ ) for all $0 \leq \alpha \leq 1$. Note that here $T_{1}^{c(\alpha)}$ is a consignment contract.

Several observations are common to the three cases. First, the contracts proposed in Theorem 2 are flexible; that is, they allow arbitrary division of objectives among the retailer and all suppliers who have a role (either production or risk-taking) in the supply chain.

Second, the three cases in the statement of Theorem 2 may overlap. For example, when supplier 1 is the least risk-averse supplier and has the same level of risk aversion as the retailer, then, besides fitting the situation in Theorem 1, it also satisfies the conditions in all cases in the above theorem. Hence in this case, these contracts proposed in all (a), (b), and (c) coordinate as well.

It is also interesting to discuss what happens in the three cases separately. In the first case, the retailer is the least risk averse. Then, it is globally optimal for the retailer to take all the risks. In this case, a properly designed quantity discount contract with supplier 1 is coordinating if other suppliers charge high enough so that it is optimal for the retailer not to order from them.

In the second case, there exists a least risk-averse supplier (supplier $m$ ) who is the least risk-averse agent in the supply chain, but it does not have the lowest production cost. As discussed earlier, it would be globally optimal in this case for this supplier to bear all risks, while the lowest-cost supplier is responsible for production. Then, a properly designed quantity discount contract between the retailer and supplier 1 and a properly designed consignment contract between the retailer and supplier $m$ is coordinating, given that other suppliers charge high enough so that the retailer will not order from them. It is important to observe that in the consignment contract between the retailer and supplier $m$, the latter charges the former a very high price per unit of item it produces. Hence, functionally, this supplier is an intermediate agent who is only responsible for the supplier to take the risk and be responsible for the production at the same time. In this case, a properly designed consignment contract between the retailer and this supplier is coordinating, again if other suppliers charge high enough.

\section{Stability}

We have identified coordinating contract profiles which depend on the level of risk aversion of the suppliers and the retailer. The contracts we propose in Theorem 2 require suppliers who should not be responsible for anything under global optimal to charge very high wholesale prices so that the retailer will not order from them. However, as these suppliers are getting nothing under the proposed contracts, they would try to propose other deals to the retailer so that the retailer will order some products from them. The question which remains is whether the proposed contracts can prevent this from happening. To analyze whether the proposed contracts are stable, we introduce two concepts to study the stability of coordinating contracts-contract core and contract equilibrium.

We first introduce the concept of contract core with the use of the core in cooperative game theory. To do so, we consider a cooperative game in which every supplier and the retailer is a player. Given the coordinating contract profile $T$ and the corresponding optimal quantity vector $\boldsymbol{q}_{s}^{*}$, the payoff of each player is its CVaR objective $U_{i}\left(\boldsymbol{q}_{s}, \boldsymbol{T}\right), i=1, \ldots, n$. Assume that there exists a value $v(C)$ associated with every coalition $C$ of players. Then, we can define a contract profile to be in the contract core if the objective value vector, $U_{i}\left(\boldsymbol{q}_{s}, \boldsymbol{T}\right)$, associated with the contract profile and the corresponding optimal quantity vector is in the core of this cooperative game.

A question that remains is how to determine the value of each coalition $v(C)$, which has been studied ever since the pioneering work of Neumann and Morgenstern (1944). The two most popular and widely accepted approaches are the $\alpha$ - and $\beta$-definitions (see 
Aumann 1967). Briefly, $v_{\alpha}(C)$ is defined as the payoff that the coalition $C$ can guarantee, while $v_{\beta}(C)$ is the payoff of coalition $C$ that agents outside the coalition cannot prevent $C$ from getting. The two definitions are equivalent when the agents' payoffs are transferrable (Aumann 1967).

Other approaches have been proposed, such as the $\gamma$-core in Chander and Tulkens (1997). However, these approaches depend on assumptions on the behaviors of agents outside the coalition, which are usually hard to justify. In particular, in a supply chain, agents outside the coalition may act cooperatively, non-cooperatively, or split into a number of coalitions. Hence we follow the traditional cooperative game approach and use the $\alpha$-core and $\beta$-core definitions as the values of the coalitions.

Following the approaches of $\alpha$-core and $\beta$-core, a coalition has to include the retailer and at least one supplier in order to build a subsystem with non-zero value. Hence, we have the following:

(a) Any coalition $C$ with a single agent has a value $v(C)=0$

(b) For any coalition $C$ without the retailer, the value of the coalition $v(C)=0$.

We now consider a coalition $C$ of agents including the retailer and at least one supplier. If $C$ is a separate supply chain, then the sum of objectives of agents in $C$ is maximized when the supply chain is coordinated. From previous analysis, this is achieved when the least risk-averse agent takes all risks while the supplier with the lowest production cost handles all the production. The sum of objectives of all agents in $C$ is the one that the coalition can guarantee. Hence, the value of the coalition is given by

$$
v(C)=\max _{q}\left(p C V a R_{\eta_{a}}(\min (q, D))-c_{i} q\right),
$$

where $a$ is the least risk-averse agent in the coalition and supplier $i$ is the supplier with the lowest production cost in this coalition.

As we have identified the value of each coalition, we are ready to provide a formal definition for the contract core as follows.

Definition 2. Let $\boldsymbol{T}\left(\boldsymbol{q}_{s}, D\right)$ be a coordinating contract profile. Then, $\boldsymbol{T}\left(\boldsymbol{q}_{s}, D\right)$ is in the contract core if there exists optimal and subgame perfect equilibrium quantities, $\boldsymbol{q}_{s}^{*}$, such that

$$
\sum_{i \in C} U_{i}\left(\boldsymbol{q}_{s}^{*}, \boldsymbol{T}\left(\boldsymbol{q}_{s}^{*}, D\right)\right) \geq v(C) \text { for all } C \subset\{0, \ldots, n\} .
$$

Under a contract profile that is in the contract core, the sum of objectives of every coalition is more than what they can achieve by working on its own. Thus, if the suppliers who have active roles (such as production or risk bearing) agree to such a contract, it is impossible for the retailer to perform better by excluding one or more of these suppliers. This way, even if the retailer renegotiates with the other suppliers, these suppliers can be assured active roles in the supply chain.

Now we turn to introduce the concept of contract equilibrium (Crémer and Riordan 1987). According to Crémer and Riordan (1987), "a set of bilateral contracts form an equilibrium if no two agents ever have an incentive to recontract, given the equilibrium behavior of other agents." Thus in our model, a contract profile $T\left(\boldsymbol{q}_{s}, D\right)$ forms a contract equilibrium if there does not exist any incentive for a supplier-retailer pair to change their contracts given the contracts of the retailer with other suppliers remain unchanged. Formally, contract equilibrium can be defined as follows.

Definition 3. A contract profile $\boldsymbol{T}\left(\boldsymbol{q}_{s}, D\right)$ and the corresponding subgame perfect equilibrium quantities $q_{s}^{*}$ is a contract equilibrium if for all $1 \leq i \leq n$, $U_{0}\left(\boldsymbol{q}_{s}^{*}, \boldsymbol{T}(\cdot)\right)+U_{i}\left(\boldsymbol{q}_{s}^{*}, \boldsymbol{T}(\cdot)\right) \geq U_{0}\left(\tilde{\boldsymbol{q}}_{s}^{*}, \tilde{\boldsymbol{T}}(\cdot)\right)+U_{i}\left(\tilde{\boldsymbol{q}}_{s}^{*}, \overline{\tilde{T}}(\cdot)\right)$ for all $\tilde{\boldsymbol{q}}_{s}^{*}$ and $\tilde{\boldsymbol{T}}(\cdot)$ such that $\tilde{T}_{j}(\cdot)=T_{j}(\cdot)$ for all $j \neq i$.

A contract equilibrium is formed if no supplierretailer pair can cooperate to increase their total profits, given the retailer's contracts with other suppliers and other suppliers' decisions remain unchanged. ${ }^{1}$ Thus, the concept of contract equilibrium studies whether there exists a supplier-retailer pair that can make a jointly profitable change to their contract after they finalize their contracts with all other agents. This is similar to the market-by-market bargaining restriction in Hart and Tirole (1990) and the pair-wise-proof concept in McAfee and Schwartz (1994). In particular, in a supply chain with one upstream firm and multiple downstream firms, the market-by-market bargaining restriction in Hart and Tirole (1990) study whether there is incentive for the upstream firm to change the contract with the downstream firm given that the contract between the upstream firm and other downstream firms remains unchanged.

There are two differences between the concept of contract core and contract equilibrium. First, the concept of contract core follows a cooperative approach, and the concept of contract equilibrium follows a more non-cooperative approach. Thus, the concept of contract core studies deviation by coalitions of any subset of agents, while the concept of contract equilibrium only guards against bilateral deviation by a supplier-retailer pair. Second, the two concepts differ in the definition of post-deviation objectives. When studying the incentives to deviate, the concept of contract core considers the objectives of the coalition if they do not contract with agents outside the coalition. On 
the other hand, the concept of contract equilibrium considers the objectives of the coalition given the existing contracts with agents outside the coalition. Thus, an observation is that contracts that form contract equilibrium may not be in the contract core and vice versa. Our objective is to identify contract profiles that are stable in the sense that they are in the contract core and at the same time constitute contract equilibrium with the optimal $q_{s}^{*}$. To do so, we first identify general conditions for contracts to be in the contract core or to constitute contract equilibrium. Then, we identify among contracts proposed in Theorem 2 those that satisfy these stability conditions.

We first analyze coordinating contract profiles that are also in the contract core. To do this, we introduce a few notions. Let

$$
S_{i}\left(c_{j}\right)=\max _{q}\left(p C V a R_{\eta_{i}}(\min (q, D))-c_{j} q\right),
$$

which is the maximum system objective function value when supplier $j$ is responsible for all production and agent $i$ (where $i=0$ denotes the retailer and $i>1$ denotes supplier $i$ ) is the risk taker.

Let

$$
S^{*}=\max _{i, j} S_{i}\left(c_{j}\right)
$$

be the optimal system total objective. Given $\boldsymbol{T}$ and the corresponding optimal production quantity vector $\boldsymbol{q}_{s}^{*}$, let $\beta_{i}=U_{s_{i}}\left(\boldsymbol{T}, \boldsymbol{q}_{s}^{*}\right) / S^{*}$ be the fraction of supplier $i^{\prime}$ s objective over the system total objective and $\beta_{0}=U_{r}\left(\boldsymbol{T}, \boldsymbol{q}_{s}^{*}\right) / S^{*}$ be the fraction of retailer's objective over the system total objective.

The next theorem identifies conditions for coordinating contracts to be in the contract core. The three cases correspond to the cases in Theorem 2. The bounds on $\beta_{i}$ reflect the bargaining powers of the agents.

\section{THEOREM 3.}

(a) Suppose the retailer is the least risk-averse agent (i.e., $\eta_{0} \leq \eta_{i}$ for all $i$ ). Then there exists $\bar{\beta}_{1}$ and $\overline{\beta_{0}}$ such that any coordinating $T \in \mathcal{T}$ is in the contract core if and only if $0 \leq \beta_{1} \leq \bar{\beta}_{1}$, $1 \geq \beta_{0}=U_{r}\left(\boldsymbol{q}_{\mathrm{s}}^{*}, \boldsymbol{T}\right) / S^{*} \geq \beta_{0^{\prime}}$ and $\beta_{i}=0$ for $i \neq 0$ or 1 . In this case, the contract profile $\boldsymbol{T}^{d(\alpha)}$ is in the contract core if and only if $\alpha \leq \bar{\beta}_{1}$.

(b) Suppose the least risk-averse supplier is also the least risk-averse agent (i.e., $\eta=\eta_{m} \leq \eta_{0}$ ) and this supplier is not supplier 1 (i.e., $m \neq 1$ ). Then there exists $\bar{\beta}_{1}, \bar{\beta}_{m}$, and $\bar{\beta}_{0}$ such that any coordinating contract profile $T \in \mathcal{T}$ is in the contract core if and only if $0 \leq \beta_{1} \leq \bar{\beta}_{1}, \quad 0 \leq \beta_{m} \leq \bar{\beta}_{m}$, $1 \geq \beta_{0} \geq \bar{\beta}_{0}$, and $\beta_{i}=0$ for $i \neq 0$ or 1 or $\mathrm{m}$. In this case, the contract profile $T^{d c(\alpha)}$ is in the contract core if and only if $\alpha \leq \bar{\beta}_{1}$ and $\gamma \geq \bar{\beta}_{0} / 1-\alpha$. (c) Suppose supplier 1 is the least risk-averse agent (i.e., $\eta=\eta_{1} \leq \eta_{0}$ ). Then, there exists $\bar{\beta}_{1}$ and $\beta_{0}$ such that any coordinating contract profile $T \in \overline{\mathcal{T}}$ is in the contract core if and only if $\beta_{1} \leq \bar{\beta}_{1}$, $\beta_{0} \geq \underline{\beta}_{0}$, and $\beta_{i}=0$ for $i \neq 0$ or 1 . In this case, the contract profile $T^{d c(\alpha)}$ is in the contract core if and only if $\alpha \leq \bar{\beta}_{1}$.

Theorem 3 implies that any coordinating contract is in the contract core as long as the agents' objectives satisfy certain conditions, which may be different depending on different cases. These conditions can be satisfied by any coordinating contracts that are flexible. The reason is that whether a coordinating contract is in the contract core depends on the objectives of the agents but not on the contract structure. Thus, the contracts proposed in Theorem 2 are also in contract cores.

It is important to discuss the bounds on the agents' share of the system objective. First, in all cases, the objective, and hence the profit, of an agent who is responsible for neither the production nor risk taking should be zero. This is reasonable, because this agent is not making any contribution to the supply chain.

In cases (a) and (b) of Theorem 3, supplier 1 is responsible for production only and it is not taking any risk of the supply chain. Observe that the bound on this supplier's share of system objective $\bar{\beta}_{1}$ is smaller when its production cost is close to the cost of supplier 2, the second lowest-cost supplier. Indeed as the difference between the production costs of these two suppliers decreases, supplier 1's bargaining power decreases and hence the fraction of system objective this supplier can claim.

In case (b), supplier $m$ is taking all the risks of the supply chain, but it is not producing anything. It is important to observe that this supplier is competing on the level of risk aversion with all other suppliers, and even with the retailer. As the level of risk of the second least risk-averse agent decreases, supplier $m^{\prime} \mathrm{s}$ bargaining power decreases, and hence its maximum claim on the fraction of system objective also decreases. In particular, this can be due to a decrease in the retailer's risk aversion level.

Consider the special case when supplier $m$ has the same level of risk aversion as the retailer. Then, its objective value is zero under any coordinating contract in the contract core. This is because this supplier will not produce anything and its only potential contribution is to take some of the risk. However, because the retailer has the same level of risk aversion, the system total objective is the same with or without this supplier. Hence, this supplier does not have any bargaining power.

Finally, in case (c), as supplier 1 is responsible for production and risk taking at the same time, its 
bargaining power is affected by both the production costs and levels of risk aversion of other agents in the supply chain.

Now we proceed to study conditions under which a contract can form contract equilibrium with the supply chain optimal decision. As it is technically challenging to identify conditions for all types of contracts, we focus on a specific set of contracts, $\mathcal{T}^{\prime}$, which is defined below.

Definition 4. A contract profile $T \in \mathcal{T}$ belongs to the subset $\mathcal{T}^{\prime} \subseteq \mathcal{T}$ if it satisfies the following conditions:

(a) $T_{i}\left(\boldsymbol{q}_{\boldsymbol{s}}, D\right)=T_{i}\left(\boldsymbol{q}_{\boldsymbol{s}}^{\prime}, D\right)$ for all $\boldsymbol{q}_{\boldsymbol{s}}$ and $\boldsymbol{q}_{s}^{\prime}$ where $q_{s_{i}}=q_{s_{i}}^{\prime}$ if supplier $i$ is not the least riskaverse agent.

(b) $T_{i}\left(\boldsymbol{q}_{s}, D\right)=T_{i}\left(\boldsymbol{q}_{s}^{\prime}, D\right)$ for all $\boldsymbol{q}_{s}$ and $\boldsymbol{q}_{s}^{\prime}$ where $q_{s_{i}}=q_{s_{i}}^{\prime}$ and $q_{0}=q_{0}^{\prime}$ if supplier $i$ is the least risk-averse agent.

(c) $T_{i}\left(\boldsymbol{q}_{s}, D\right)$ is non-decreasing in $D$ for all $\boldsymbol{q}_{s}$ for all $i$ and $p \min \left(q_{0}, D\right)-\sum_{i=1}^{n} T_{i}\left(\boldsymbol{q}_{\boldsymbol{s}}, D\right)$ is nondecreasing in $D$.

The conditions in Definition 4 are very general. The first condition in Definition 4 requires that the payment between the retailer and any supplier $i$ who is not the least risk-averse agent depend only on the quantity produced by supplier $i$, which is reasonable as a supplier cannot easily verify how much the retailer orders from other suppliers. The second condition requires that the payment between the retailer and the supplier who is the least risk-averse agent in the supply chain can depend on the total quantity available in the market. This may be less desirable than allowing the payment between the least risk-averse supplier and the retailer to be dependent on the quantity produced by each individual supplier, but we argue that it is enough for studying coordinating contracts because only the supplier with the lowest production cost will receive a strictly positive order quantity from the retailer. Finally, the last condition implies that both the payment from the retailer to each supplier and the retailer's profit have to be non-decreasing in the market demand. This condition is satisfied for most practical contracts, such as buyback contracts, revenue sharing contracts, and linear rebate contracts.

For the rest of this section, we focus on, $\mathcal{T}$, the set of contracts that satisfy these three conditions. The next theorem characterizes conditions under which contracts in $\mathcal{T}^{\prime} \subseteq \mathcal{T}$ can form contract equilibrium with the supply chain optimal decision.

THeOREM 4. Let $T \in \mathcal{T}^{\prime}$ be a coordinating contract profile. Then $\mathbf{T}$ forms a contract equilibrium with the supply chain optimal decision profile $\boldsymbol{q}_{s}^{*}$ if the following conditions hold:
(a) $\boldsymbol{q}_{s}^{*}$ maximizes the retailer's objective, $U_{0}\left(\boldsymbol{T}, \boldsymbol{q}_{s}\right)$, and $q_{s_{1}}^{*}$ maximizes supplier $1^{\prime} s$ objective, $U_{1}\left(\boldsymbol{T}, \boldsymbol{q}_{s}\right)$.
(b) If supplier $m$ is the least risk-averse agent, that is, $\eta_{m} \leq \eta_{i}$ for all $i$, and $T_{m}\left(q_{s_{m}}, q_{0}\right) \neq T_{m}\left(q_{s_{m}}, q_{0}^{*}\right)$ for some $q_{s_{m}}$ and $q_{0}$, then $q_{s_{m}}^{*}$ and $q_{0}^{*}$ maximize $U_{m}\left(\boldsymbol{T}, \boldsymbol{q}_{s}\right)$.
(c) $\operatorname{CVaR}_{\eta}\left(T_{1}\left(q_{s_{1}}, \cdot\right)\right)-\operatorname{CVaR}_{\eta}\left(T_{1}\left(q_{s_{1}}^{\prime}, \cdot\right)\right) \leq c_{i}\left(q_{s_{1}}-q_{s_{1}}^{\prime}\right)$ where $\eta=\min \left(\eta_{0}, \eta_{i}\right)$ for all $q_{s_{1}} \geq q_{s_{1}}^{\prime} \geq 0$ for all $i>1$.

Theorem 4 identifies several sufficient conditions for a coordinating contract profile in the set $\mathcal{T}$ to be a contract equilibrium with the supply chain optimal decision. Condition (a) requires that the optimal production quantity is optimal for the retailer and for the lowest-cost supplier. That is, no supplier is producing less than what is ordered by the retailer, and, in addition, the lowest-cost supplier would not produce more even if the retailer had ordered more. There are two compliance regimes in contracts, voluntary compliance and forced compliance (see Cachon 2003 for detail). Under voluntary compliance, a supplier can produce less than the quantity ordered by the retailer. Under forced compliance, a supplier has to deliver the exact quantity ordered by the supplier. Condition (a) in Theorem 4 implies that the contract has to be coordinating under forced compliance also (although the model we study assumes only voluntary compliance), and, in addition, the lowest-cost supplier will produce the system optimal quantity if he can decide how much to produce freely.

Condition (b) suggests that the optimal total supply chain quantity has to be optimal for the supplier who is the least risk-averse agent. Under a coordinating contract, this supplier is bearing risks for the whole supply chain. The larger the total quantity, the higher the risk this supplier has to bear. Hence, condition (b) suggests that the level of risk that this supplier has to bear is optimal for him.

Condition (c) in Theorem 4 requires the marginal cost of ordering from the lowest-cost supplier to be always smaller than the production cost of any other supplier. No supplier is willing to accept a contract in which the payment cannot cover its production cost. Therefore, even if the retailer renegotiates its contract with a supplier who does not have the lowest cost, the retailer will not change its ordering decision and order less from the lowest-cost supplier.

It is also important to note that that there is no direct relationship between the conditions in Theorem 4 and the share of objectives of the agents. For example, suppose we add a fixed transfer payment to a 
contract profile. The transfer payment changes the objective shares of the agents, but it does not influence whether the contract profile satisfies the conditions in Theorem 4 or not. Therefore, Theorem 4 implies that whether a coordinating contract can form a contract equilibrium with the supply chain optimal decision depends on the contract structure or how the payment changes with the order quantity. This is in contrast with Theorem 3, which implies that whether a coordinating contract profile lies in the contract core depends solely on the objective share. Thus, combining Theorems 3 and 4, we can identify among contracts in Theorem 4 those that are stable in terms of the share of objectives and contract structure.

\section{Corollary 1.}

(a) The risk-aversion level of the retailer is less than or equal to the risk-aversion levels of all suppliers, that is, $\underline{\eta}=\eta_{0} \leq \eta_{i}$ for all $i$. Then $T^{d(\alpha)}$ is a coordinating contract profile in the contract core and forms a contract equilibrium with the supply chain optimal strategy profile $q_{s}^{*}$ for all $0 \leq \alpha \leq$ $\min \left(1-\left(S_{0}\left(c_{2}\right)\right) /\left(S^{*}\right),\left(c_{2}-c_{1}\right) /\left(p-c_{1}\right)\right)$.

(b) Suppose the risk-aversion level of the retailer is higher than or equal to the level of the least riskaverse supplier, who does not have the lowest production cost, that is, $\eta=\eta_{m} \leq \eta_{0}$ but $c_{m} \neq c_{1}$. Then $\boldsymbol{T}^{d c(\alpha)}$ is a coordinating contract profile in the contract core and forms a contract equilibrium with the supply chain optimal strategy profile $q_{s}^{*}$ for all $0 \leq \alpha \leq \min \left(1-\left(S_{m}\left(c_{2}\right) / S^{*}\right),\left(c_{2}-c_{1}\right)\right.$ / $\left.\left(p-c_{1}\right)\right)$ and $\frac{1}{(1-\alpha)} \max \left(\max _{j \neq 1, m} S_{j}\left(c_{2}\right) / S^{*}\right.$, $\left.\max _{j \neq m}\left(S_{j}\left(c_{1}\right) / S^{*}\right)-\alpha\right) \leq \gamma \leq 1$.

(c) Suppose the supplier with the lowest production cost has the least risk-averse level among all agents, that is, $\eta=\eta_{1} \leq d_{r}$. Then $T^{c(\alpha)}$ is a coordinating contract profile in the contract core and forms a contract equilibrium with the supply chain optimal strategy profile $q_{s}^{*}$ for all $0 \leq \alpha \leq \min$ $\left(1-\max _{j \neq 1}\left(S_{j}\left(c_{2}\right) / S^{*}\right),\left(c_{2}-c_{1}\right) /\left(p-c_{1}\right)\right)$.

In the contracts proposed in Corollary 1, the objective value of the retailer and all suppliers who do not have the lowest cost are bounded because of the conditions for contract core in Theorem 3 There are two upper bounds for the objective value of the lowest-cost supplier. One is due to the condition for contract core, while the other bound is to make sure the contract structure allows the contract to form a contract equilibrium with the supply chain optimal decision. Hence, not all the objective share allocations in the contract core can be achieved by the contracts proposed in Corollary 1 . To allow for more flexible objective share, a fixed payment from the retailer to the lowest-cost supplier can be added to the contract profile in each case.
The contracts will be able to form contract equilibrium with the supply chain optimal decision regardless of the size of the fixed payment. However, to make sure that the contracts are in the contract core, the fixed payment should be small enough so that the overall objective of the lowest-cost supplier is smaller than the upper bound stated in Theorem 3 .

\section{Conclusions and Discussion}

In this article, we study a supply chain with a single risk-averse retailer and multiple risk-averse suppliers, all of whom maximize the CVaR of their profits.

We show that the supply chain is coordinated when the lowest-cost supplier produces all the products while the least risk-averse agent (who may be the retailer or one of the suppliers) bears all risks. We identify coordinating contracts for the supply chain in different cases based on the relative risk-aversion levels of the agents and the production costs of the suppliers. These contracts allow flexible objective sharing among all the agents, but some of them may not be stable.

We also introduce the concepts of contract core and contract equilibrium to study the stability of coordinating contracts. Using the concept of the contract core, we characterize conditions for contracts to be stable from a cooperative game perspective. Under these conditions, the objectives of the agents are bounded depending on their relative bargaining powers (i.e., products costs and risk-aversion levels). Then, using the concept of the contract equilibrium, we identify conditions for contracts to be stable from a non-cooperative game perspective. These conditions are not related to the objectives of the agents, but to the structure of the contracts. Using concepts of contract core and contract equilibrium, we reduce the set of coordinating contracts to a smaller set that are stable in terms of both the objectives of the agents and also the contract structure.

Our results are sensitive to the risk-aversion levels of the agents only to a certain extent. First, changes in the risk-aversion levels of agents who are not the least risk averse have no impact of any on the results as long as none of these agents become the least risk averse. Second, the risk-aversion level of the least risk-averse agent does not affect the coordinating and stable contract form (such as consignment and quantity discount). It only affects the contract parameters and the conditions under which these contracts are in the contract core and form contract equilibrium.

The concepts of contract core and contract equilibrium defined here are quite general and can be applied to analyze other complex supply chains as well. The concept of contract core, however, has a few limitations. First, as mentioned before, we use the 
$\alpha$-core concept as the definition of contract core contract, which may be conservative in calculating the value of each coalition of agents. This contract core contract concept allows us to exclude some "non-stable" coordinating contracts, but the set of contract core contracts may still be very broad. One possible remedy is to use other core concepts from cooperative game theory when finding the set of contract core contracts.

In many supply chains, different agents are competing against each other and there is no single agent who has the power to propose take-it-or-leave-it contracts to all its partners. In these cases, the principalagent model may not be appropriate. Studies on the stability of coordinating contracts can help us identify contracts that the agents could adopt. There are several possible directions for future extensions. First, the concepts of contract core and contract equilibrium can be extended to identify stable coordinating contracts in other supply chain settings (such as one with multiple retailers), in service outsourcing or in other situations (such as financial contracts). For instance, we can use these concepts to analyze a supply chain with multiple retailers and a single supplier. Interestingly, in this case, when all agents are risk averse with CVaR as the risk measure, we can still show that supply chain optimality is achieved when the least riskaverse agent bears the entire risks. Of course, the specific form of (stable) coordination contract will depend on the form of competition among the retailers.

Second, contract core and contract equilibrium are only two of the many approaches to study stability of contracts. One possible future direction is to analyze the advantages and disadvantages of these approaches, and to identify other approaches to study the stability of contracts. We believe that the stability of coordinating contracts presents a fruitful direction for further research.

\section{Acknowledgments}

The authors thank department editor Panos Kouvelis, an anonymous senior editor, and two anonymous reviewers for helpful suggestions. This research is supported in part by the Singapore-MIT Alliance, by NSF DMI-9732795, DMI0085683, DMI-0245352, CMMI-0653909, and CMMI-0926845 ARRA, and by RGC GRF 619508.

\footnotetext{
Note

${ }^{1}$ Our definition assumes that the retailer can change $q_{s}$ arbitrarily when changing her contract with a supplier. This is true in the case of forced compliance when the retailer has total control over $q_{s}$. In this case, the condition in our definition is sufficient and necessary. In the voluntary compliance case, the retailer has a certain degree of control over $q_{s}$ (because $q_{s} \leq q_{r}$ ), but it may only be able to choose from a certain set of $\boldsymbol{q}_{s}$. In this case, the condition in our definition is still sufficient but may not be necessary.
}

\section{References}

Agrawal, V., S. Seshadri. 2000. Risk intermediation in supply chains. IIE Trans. 32(9): 819-831.

Ahmed, S., U. Çakmak, A. Shapiro. 2007. Coherent risk measures in inventory problems. Eur. J. Oper. Res. 182(1): 226-238.

Allais, M. 1953. Le comportement de l'homme rationnel devant le risque critique des postulats et axiomes de l'ecole americaine. Econometrica 21: 503-546.

Amihud, Y., B. Lev. 1981. Risk reduction as a managerial motive for conglomerate mergers. Bell J. Econ. 12(2): 605-617.

Anupindi, R., Y. Bassok, E. Zemel. 2001. A general framework for the study of decentralized distribution systems. Manuf. Serv. Oper. Manag. 3(4): 349-368.

Artzner, P., F. Delbaen, J. M. Eber, D. Heath. 1999. Coherent measures of risk. Math. Finance 9(3): 203-228.

Aumann, R. 1967. A survey of cooperative games without side payments. M. Shubik, ed. Essays in Mathematical Economics in Honor of Oskar Morgenstern. Princeton University Press, Princeton, NJ, 3-27.

Bernstein, F., L. Marx. 2006. Reservation profit levels and the division of supply chain profits. Working paper, Duke University, Durham, NC.

Bucay, N., D. Rosen. 1999. Credit risk of an international bond portfolio: A case study. ALGO Res. Quart. 2(1): 9-29.

Cachon, G. 2003. Supply chain coordination with contracts. T. De Kok, S. Graves, eds. Handbooks in Operations Research and Management Science: Supply Chain Management. Elsevier, Amsterdam, 229-340.

Chander, P., H. Tulkens. 1997. The core of an economy with multilateral externalities. Int. J. Game Theory 26: 379-401.

Chen, W., M. Sim. 2006. Goal driven optimization. Oper. Res. 57(2): 342-357.

Chen X., M. Sim, D. Simchi-Levi, P. Sun. 2007. Risk aversion in inventory management. Oper. Res. 55(5): 828-842.

Chen, Y., M. Xu, Z. Zhang. 2009. A risk-averse newsvendor model under the CVaR decision criterion. Oper. Res. 57(4): 10401044.

Crémer, J., M. Riordan. 1987. On governing multilateral transactions with bilateral contracts. RAND J. Econ. 18(3): 436-451.

de Fontenay, C., J. Gans. 2005. Vertical integration in the presence of upstream competition. RAND J. Econ. 36(3): 544-572.

Dowd, K. 1998. Beyond Value at Risk. Wiley, New York.

Duffie, D., J. Pan. 1997. An overview of value at risk. J. Derivatives 4(3): 7-49.

Embrechts, P., S. Kluppelberg, T. Mikosch. 1997. Extremal Events in Finance and Insurance. Springer Verlag, New York.

Gan, X., S. Sethi, H. Yan. 2004. Coordination of a supply chain with risk-averse agents. Prod. Oper. Manag. 13(2): 135-149.

Gollier, C. 2004. The Economics of Risk and Time. The MIT Press, Cambridge, MA.

Gurnani, H., M. Shi. 2006. A bargaining model for a first-time interaction under asymmetric beliefs of supply reliability. Manage. Sci. 52(6): 865-880.

Hart, O., J. Tirole. 1990. Vertical integration and market foreclosure. Brookings Papers Econ. Activity special issue 205-286.

Hull, J. 2007. VAR versus Expected Shortfall. Rick Magazine, March 1. Available at http://www.risk.net/risk-magazine/technicalpaper/1506669/var-versus-expected-shortfall (accessed date May 4, 2013)

Jensen, M., W. Meckling. 1976. Theory of the firm: Managerial behavior agency costs and ownership structure. J. Financial Econ. 3(4): 305-360.

Jorion, P. 1997. Value at Risk. McGraw-Hill, New York. 
Chen, Shum, and Simchi-Levi: Stable and Coordinating Contracts for a Supply Chain Production and Operations Management 23(3), pp. 379-392, (c) 2013 Production and Operations Management Society

Lanzillotti, R. F. 1958. Pricing objectives in large companies. Am. Econ. Rev. 48(5): 921-940.

McAfee, R., M. Schwartz. 1994. Opportunism in multilateral vertical contracting: Nondiscrimination exclusivity and uniformity. Am. Econ. Rev. 84(1): 210-230.

Mulvey, J., H. Erkan. 2005 Applying CVaR for decentralized risk managmeent of financial companies. J. Banking Finance 30: 627-644.

Nagarajan, M., Y. Bassok. 2002. A bargaining framework in supply chains: The assembly problem. Manage. Sci. 54(8): 1482-1496.

Nagarajan, M., G. Sosic. 2007. Stable farsighted coalitions in competitive markets. Manage. Sci. 53(1): 29-45.

Nagarajan, M., G. Sosic. 2009. Coalition stability in assembly models. Oper. Res. 57(1): 131-145.

O'Brien, D., G. Shaffer. 1992. Vertical control with bilateral contracts. RAND J. Econ. 23(3): 299-308.

Payne, J. W. 2005. It is whether you win or lose: The importance of the overall probabilities of winning or losing in risky choice. J. Risk Uncertainty 30(1): 5-19.

Payne, J. W., D. J. Laughhunn, R. Crum. 1980. Translation of gambles and aspiration level effects in risky choice behavior. Manage. Sci. 26(10): 1039-1060.

Rajgopal, S., T. Shevlin. 2002. Empirical evidence on the relation between stock option compensation and risk taking. J. Account. Econ. 33: 145-171.
Rockafellar, R., S. Uryasev. 2000. Optimization of conditional value-at-risk. J. Risk 2(3): 21-42.

Rockafellar, R., S. Uryasev. 2002. Conditional value-at-risk for general loss distributions. J. Banking Finance 26(7): 1443-1471.

Shapira, Z. 1994. Risk Taking: A Managerial Perspective. Russell Sage Foundation, New York.

Spulber, D. 1985. Risk sharing and inventories. J. Econ. Behav. Org. 6(1): 55-68.

Street, A. 2010. On the conditional value-at-risk probabilitydependent utility function. Theory Decis. 68(1-2): 49-68.

Tomlin, B., Y. Wang. 2005. On the value of mix flexibility and dual sourcing in unreliable newsvendor networks. Manuf. Serv. Oper. Manag. 7(1): 37-57.

Topaloglou, N., H. Vladimirou, S. Zenios. 2002. CVaR models with selective hedging for international asset allocation. J. Banking Finance 26(7): 1535-1561.

von Neumann, J., O. Morgenstern. 1944. Theory of Games and Economic Behavior. Princeton University Press, Princeton, NJ.

\section{Supporting Information}

Additional Supporting Information may be found in the online version of this article:

Appendix S1: Proofs 